\title{
Interchangeability of Sodium and Potassium Result Values of Arterial Blood Gas with Laboratory Analyzer: Narrative Review
}

\author{
${ }^{1}$ Yasemin Ustundağ, ${ }^{2}$ Kağan Huysal, ${ }^{3}$ Şeyda E Ozgunay, ${ }^{4}$ Ali R Turkoğlu
}

\begin{abstract}
The major extracellular electrolytes, sodium, and potassium are often requested together and form a large percentage of the requested tests in routine clinical chemistry laboratories. Two types of devices that use direct and indirect ion-selective electrode (ISE) methods are used in hospitals for electrolyte measurements: blood gas analyzers (BGA), which use direct ISE technology, and the indirect ISE method, which is often used in a central-laboratory autoanalyzer (AA).
\end{abstract}

We aimed to summarize the current scientific knowledge based on whether the electrolyte test results, using $\mathrm{Na}$ and $\mathrm{K}$ test results obtained with $B G A$ and an $A A$, can be used interchangeably.

We searched Medline (PubMed), Google Scholar, and Web of Science up to $31^{\text {st }}$ March 2018. In addition, references of the included studies were also examined.

Fourteen studies with a risk of bias were included in the analysis. Limits of agreement differences were variable among BGA and AA sodium and potassium test results in clinical practice.

The results of both BGA and AA measures should not be used interchangeably under the assumption that they are equivalent to each other.

Keywords: Analyser, Ion-selective electrode, Potassium, Sodium.

How to cite this article: Ustundağ Y, Huysal K, Ozgunay ŞE, Turkoğlu AR. Interchangeability of Sodium and Potassium Result Values of Arterial Blood Gas with Laboratory Analyzer: Narrative Review. Indian Journal of Critical Care Medicine, January 2019;23(1),35-42.

Source of support: Nil

Conflict of interest: None

\footnotetext{
${ }_{1}^{1}{ }^{4}$ Researchers

1,2Department of Clinical Biochemistry, University of Health Sciences, Bursa Yukseklhtisas Training and Research Hospital, Bursa 16290, Turkey

${ }^{3}$ Department of Anestesiology and Reanimation, University of Health Sciences, Bursa Yukseklhtisas Training and Research Hospital, Bursa 16290, Turkey

${ }^{4}$ Department of Urology, University of Health Sciences, Bursa Yukseklhtisas Training and Research Hospital, Bursa 16290, Turkey

Corresponding author: Yasemin Ustundağ,Researchers, Department of Clinical Biochemistry, University of Health Sciences, Bursa Yukseklhtisas Training and Research Hospital, Bursa 16290, Turkey, Phone:+905324823692, e-mail: yaseminbudak2000@yahoo.com
}

\section{INTRODUCTION}

The major extracellular electrolytes, sodium, and potassium are often requested together and form a large percentage of requested tests in routine clinical chemistry laboratories.

Sodium is responsible for the osmotic pressure of the extracellular fluid, and the physiological level in serum is $135 \mathrm{mmol}$ to $145 \mathrm{mmol} / \mathrm{L}^{1}$ Potassium is the major cation in intracellular fluid and, despite playing a physiological role in such processes as a contraction of skeletal muscles, it is necessary for normal cell function. ${ }^{1}$ The physiological serum concentration of potassium is 3.5 to $5.0 \mathrm{mmol} / \mathrm{L} .{ }^{1}$

Electrolyte disorders constitute a significant proportion of emergency department (ED) visits and are also common in the intensive care unit (ICU) patients and critically ill patients. ${ }^{2-6}$ Hyponatremia, defined as a sodium concentration $<135 \mathrm{mmol} / \mathrm{L}$, is the most common electrolyte abnormality encountered in the emergency room and ICU and can lead to serious neurological complications. ${ }^{3,4}$ Hyperkalemia, which occurs with potassium higher than $5.0 \mathrm{mmol} / \mathrm{L}$, is a common electrolyte disorder leading to morbidity and mortality due to cardiac dysrhythmia, especially in ED patients. ${ }^{5}$

Because abnormal serum sodium and potassium levels are associated with mortality and morbidity, it is extremely important for patients to be diagnosed quickly and to start treatment early. ${ }^{6}$

\section{Laboratory Diagnosis of Electrolyte Disorders}

Electrolytes are routinely measured by either direct or indirect ion-selective electrodes (ISE). The principle of the method is based on the determination of the electromotive power (potential) changes occurring between the measuring electrode and the reference electrode, whereas the ion to be measured interacts with the ISE membrane.

Two different types of devices using direct and indirect ISE methods are used in hospitals for electrolyte measurements. Blood gas analyzers (BGA) use direct ISE technology that measures electrolytes in undiluted sample types. Devices based on the indirect ISE method are often used in a high-efficiency central laboratory automated analyzer (AA). Before measuring electrolyte 
concentrations with the indirect ISE method, the same diluent volume is used by estimating the amount of dilution by the expected solid fraction (7\%). However, if the solid fraction is increased, as, during hyperproteinemia, the measured ion concentration is underestimated because of the higher dilution. ${ }^{8}$

Electrolyte values of the venous blood samples can be obtained after an average of 60 minutes in the AA in routine clinical chemistry laboratories. In the meantime, treatments depending on electrolyte values are required to be performed immediately, and are either done blindly or are delayed. ${ }^{9}$ In addition, the result times are even longer when the samples are hemolyzed, lipemic, inadequate, or lost, and while the devices are in the process of calibration. BGAs are especially advantageous in such places as emergency service units and ICUs because all measurement results are available in as little as 2 minutes.

However, in routine clinical practice, BGA electrolyte findings are generally used to support diagnoses; that leads to a comparison of these device outputs to the AA results. ${ }^{10,12}$ With the observation of differences between the two results (BGAversus AA), even if samples are taken at the same time, physicians are often faced with the question of which test result to use in patient treatment, especially when therapy is to be initiated or frequent measurements are made to guide treatment.

We aimed to find whether the electrolyte test results using $\mathrm{Na}$ and $\mathrm{K}$ test results obtained with BGA and AA could be used interchangeably.

\section{METHOD}

This article is neither a systematic review nor a metaanalysis. We searched Medline (Pubmed), Google Scholar, and Web of Science for English only in humans up to 31st March 2018; using the query 'blood gas analyzer or autoanalyzer in combination with sodium and/or potassium'. To eliminate as much unrelated research as possible, we determined that keywords must appear in the title or abstract. In addition, to include as many related studies as possible, references of the included studies were also examined. We then selected the publications with potential practical usefulness. We kept studies of adults but not children.

We excluded studies that use correlation and regression analysis, or the difference between the averages instead of Bland-Altman methods. The Bland-Altman plot quantifies the bias and a range of agreement within which $95 \%$ of the differences of the second method (as compared to the first one) fall. The Bland-Altman method objectively measures the differences between measurement techniques. ${ }^{12,13}$

The acceptability criteria of interchangeability of results were derived from The United States Clinical Laboratory Improvement Amendments (US CLIA) guidelines, which state that $95 \%$ of results should fall within $0.5 \mathrm{mmol} / \mathrm{L}$ for potassium levels and $4 \mathrm{mmol} / \mathrm{L}$ for measured sodium levels to assess the intralaboratory quality of clinical chemistry tests. ${ }^{13,14}$

\section{RESULTS}

There are some methodological problems when comparing BGA and AA electrolyte results. For example, some studies used the results of the heparinized arterial sample is plasma and the venous sample is serum the patient's database retrospectively, whereas others compared prospective sample results. As this may account for some of the differences.

\section{A. Some prospective studies investigate whether the electrolyte test results using arterial whole blood versus arterial plasma or a serum specimen can be used interchangeably.}

In some of these prospective studies, the mean bias for $\mathrm{Na}$ was 1.3 to $1.7 \mathrm{mmol} / \mathrm{L}$ and for $\mathrm{K} 0.2$ to $0.3 \mathrm{mmol} / \mathrm{L}$, which are acceptable ranges using analytical goals defined by CLIA. ${ }^{15-17}$ However, $95 \%$ limits of agreement (LOA) were between -9.4 and12.6 $\mathrm{mmol} / \mathrm{L}$ for $\mathrm{Na}$ and -0.58 and $1.24 \mathrm{mmol} / \mathrm{L}$ for $\mathrm{K}$, and interchangeable use is unacceptable. ${ }^{15,16}$

In these studies, the devices of different manufacturers were compared (Table 1). Yilmaz et al. compared the results of the Abbott C 8000 Architect AA (Abbott Diagnostics, Abbott Laboratories, North Chicago, Illinois, USA) and Siemens Rapid Point 500BGA(Siemens Healthcare Diagnostics, Inc. Tarrytown, NY, USA) ${ }^{15}$ King et al. compared the results of the electrolytes with a Hitachi 717AA (Boehringer Mannheim, Lewes, West Sussex, UK) and a Radiometer ABL 505 BGA(Radiometer, Crawley, West Sussex, UK). ${ }^{16}$

Allardet-Servent et al. demonstrated that $48 \%$ of the differences between the electrolyte test results of the two analyzers-an AU 580 AA(Beckman Coulter, Brea, CA, USA) and a RAPID Point 500 BGA(Siemens Healthcare Diagnostics Inc., Tarrytown, NY, USA)—were due to changes in the serum protein level. ${ }^{17}$

Chacko et al. compared the sodium results of whole blood and serum samples; the mean bias was -4.07 $\mathrm{mmol} / \mathrm{L}$ and $95 \%$ LOA -8.8 to $0.7 .^{18}$ They used a GEM 3000 BGA (Instrumentation Laboratory, Werfen, Italy) and an Olympus AU2700 AA (Olympus Optical Company, Ltd., Japan). The mean difference in potassium values was $-0.3 \mathrm{mmol} / \mathrm{L}$ and $95 \%$ LOA -0.72 to $+0.13 \mathrm{mmol} / \mathrm{L}$. However, individual differences were clinically significant, especially at low potassium levels $(<3.0 \mathrm{mmol} / \mathrm{L})$, 


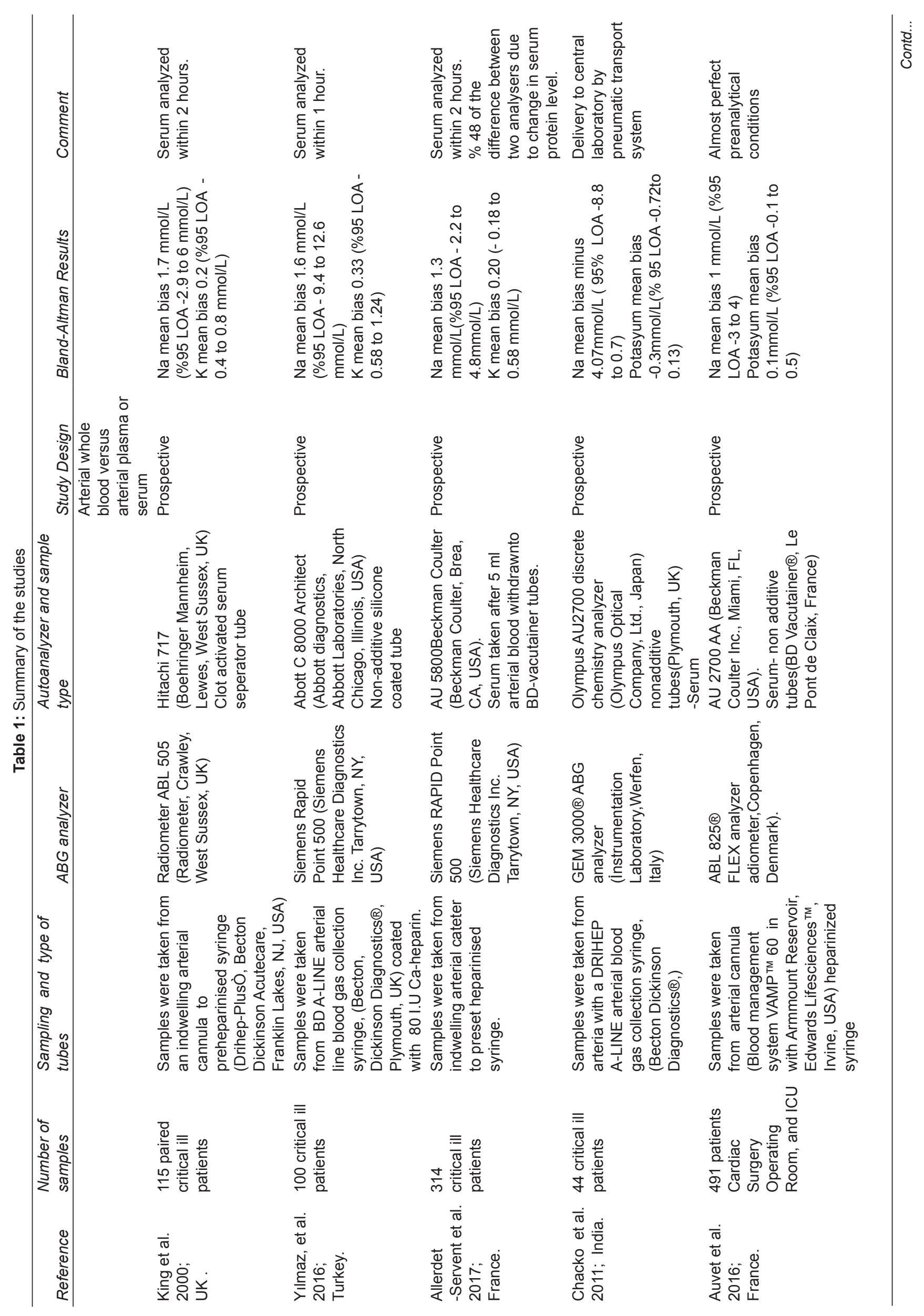




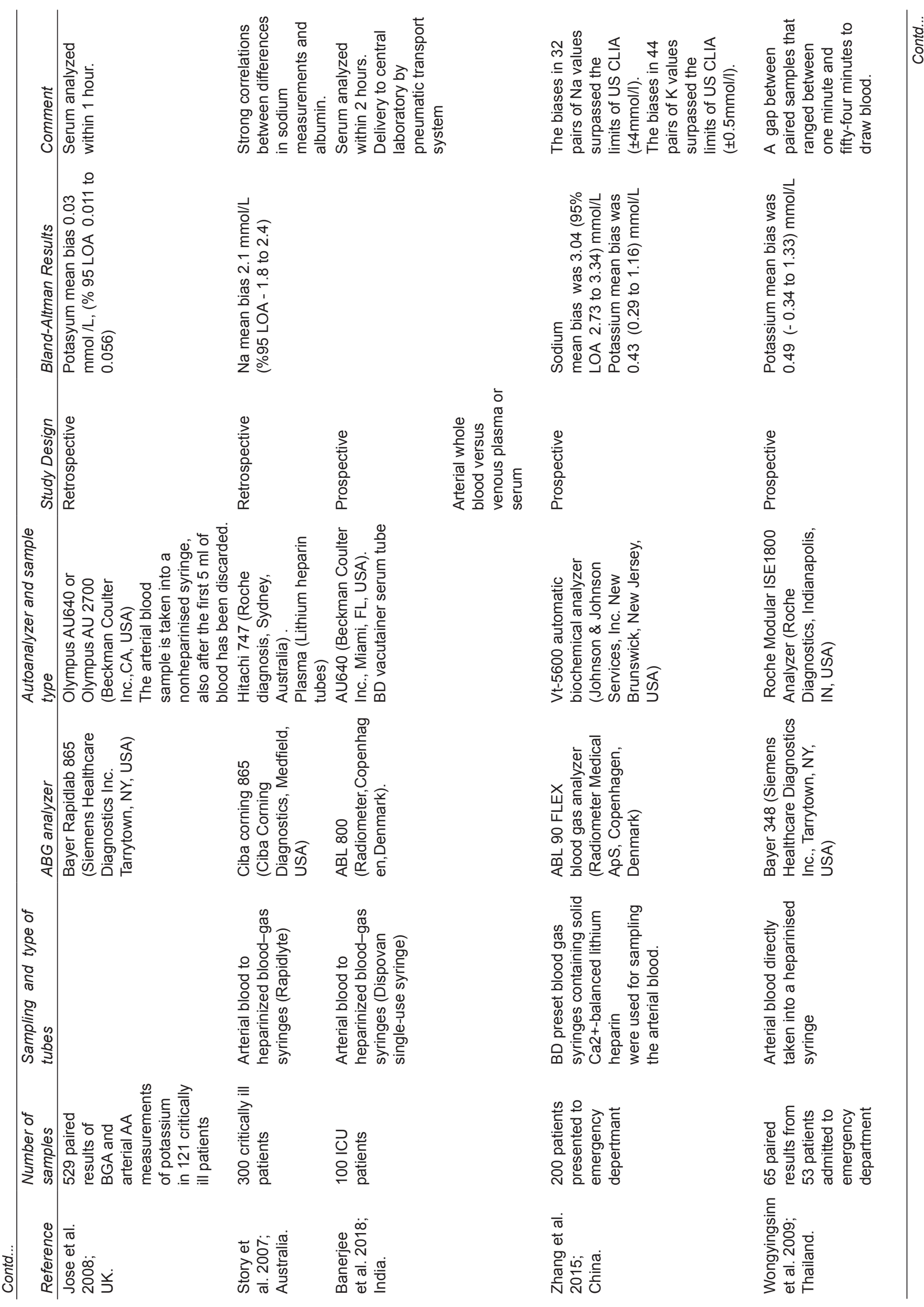




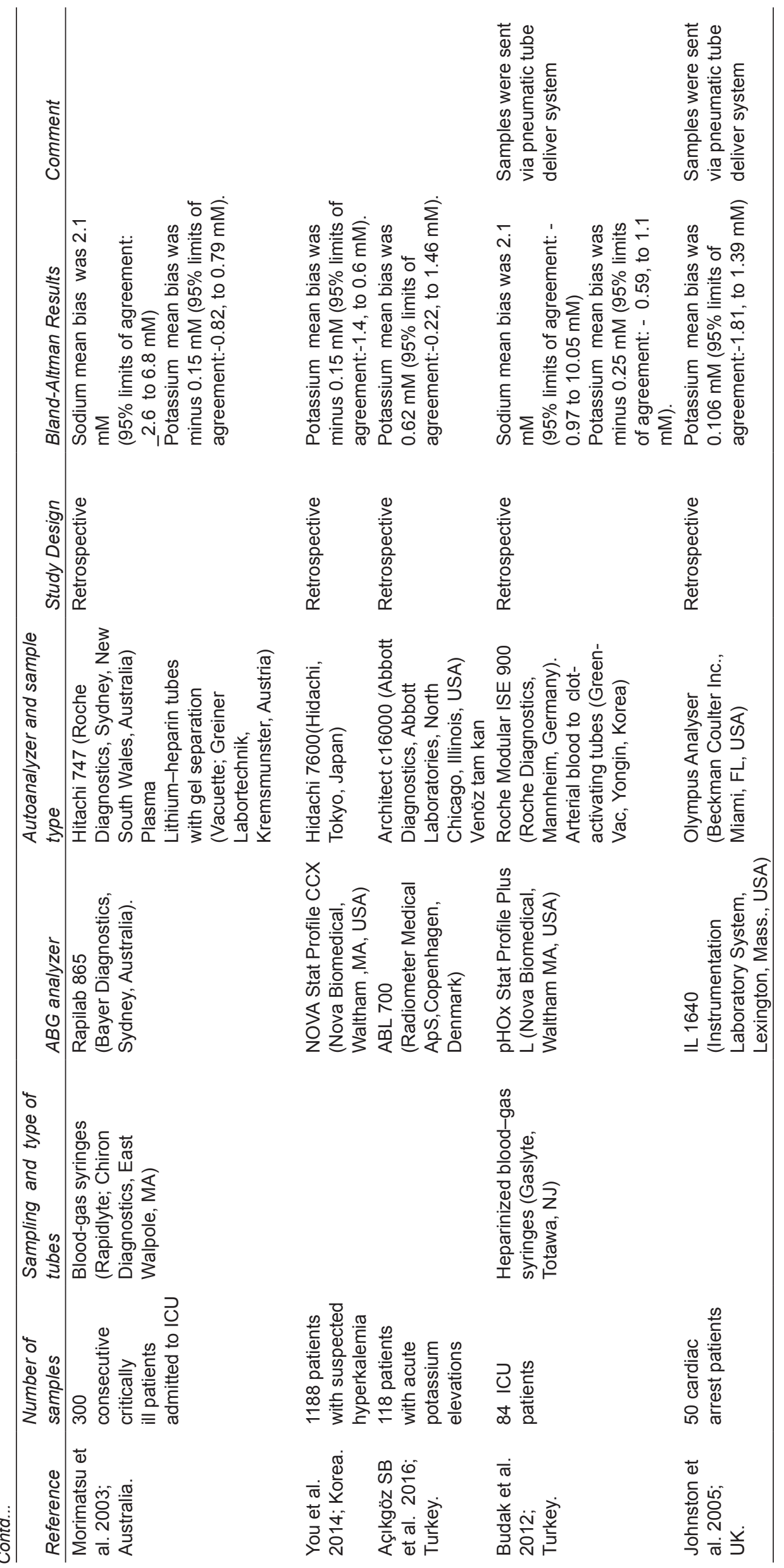


and they suggested that delivery to the central laboratory by a pneumatic transport system may lead to hemolysis. ${ }^{18}$

Auvet et al. compared 491 paired whole blood and plasma sample electrolyte levels with ABL $825^{\circledR}$ FLEX BGA (Radiometer, Copenhagen, Denmark) and AU2700 AA(Beckman Coulter Inc., Miami, FL, USA) devices. Because preanalytical conditions were near-perfect, the bias of the results obtained for potassium was 0.1 (95\% LOA 0.1-0.5), and the bias for sodium was 1 (95\% LOA minus 3 to 4 ); both results are interchangeable. ${ }^{19}$

In a recent study, Banerjee et al. compared ABL 800 (Radiometer, Copenhagen, Denmark) with an AU640 AA (Beckman Coulter Inc., Miami, FL, USA) analyzers to find a correction factor for the appropriation of the $A B G$ value with the AA to minimize all errors. They concluded that a correction factor should be determined individually for each hospital. ${ }^{20}$

\section{B. Some of the studies were retrospective, comparing the levels of electrolytes using arterial whole blood versus arterial plasma or serum}

Jose et al. compared critical electrolytes run on a Bayer Rapidlab 865 BGA (Siemens Healthcare Diagnostics Inc., Tarrytown, NY, USA) and an Olympus AU640 AAor an Olympus AU2700 AA (Beckman-Coulter, Inc., Fullerton, CA, USA). The difference between the potassium values of the two methods is $0.03 \mathrm{mmol} / \mathrm{L}$ and $95 \%$ LOA 0.011 to 0.056 . The Bland-Altman statistical method shows that even in hypokalemia and hyperkalemia, 95\% of the patient results bias is less than $0.5 \mathrm{mmol} / \mathrm{L}^{21}$

Story et al.evaluated electrolytes with albumin levels and demonstrated that if the plasma albumin level was above $40 \mathrm{~g} / \mathrm{L}$, the bias was 0 , and the indirect ISE Na value was found to be higher in hypoalbuminemia patients. ${ }^{22}$

\section{Some of the prospective studies compare the levels of electrolytes using arterial whole blood versus venous serum or plasma.}

The study of Zhang et al. prospectively compared arterial and venous blood $\mathrm{Na}$ and $\mathrm{K}$ results obtained with an ABL 90 FLEX BGA (Radiometer Medical ApS, Copenhagen, Denmark) and a VT-5600AA (Johnson and Johnson Services, Inc., New Jersey, USA). The mean difference between the two devices for sodium was 3.04, and $95 \%$ LOA was minus 1.24 to $7.31 \mathrm{mmol} / \mathrm{L}$. The paired test result biases of 32/50 were higher than the values accepted by the US CLIA. The average bias for potassium was $0.43 \mathrm{mmol} / \mathrm{L}$ (95\% LOA minus $0.29-1.16 \mathrm{mmol} / \mathrm{L})$. The 44/50 pairs of values exceeded the acceptable range for US CLIA $(0.5 \mathrm{mmol} / \mathrm{L}){ }^{23}$

Wongyingsinn et al. studied whole blood electrolyte levels with a Bayer 348BGA (Bayer Diagnostics, Siemens Healthcare Diagnostics Inc., Tarrytown, NY, USA) and venous blood with a Roche Modular ISE 1800 AA (Roche Diagnostics, Indianapolis, IN, USA). The mean difference between the two methods was $0.49 \mathrm{mmol} / \mathrm{L} \mathrm{(95 \%} \mathrm{CI} \mathrm{of}$ LOA 0.893-0.943). However, this difference is explained by the range of 1-54 minutes for taking blood from the patient. $^{24}$

\section{Retrospective studies comparing the levels of electrolytes using arterial whole blood versus venous serum or plasma.}

In retrospective studies comparing the arterial whole blood and venous serum or plasma results, the mean bias for sodium was found to be 4.9 to $2.1 \mathrm{mmol} / \mathrm{L}$, and the LOA was minus 0.97 to $10.05 \mathrm{mmol} / \mathrm{L} .{ }^{11,25}$ With the comparison of potassium, Bland-Altman AA, and BGAdata, the mean bias was 0.106 to $0.4 \mathrm{mmol}, 14,28,29$ 95\% LOA-1.4 and $1.394 \mathrm{mmol} / \mathrm{L}$, and the two devices were not interchangeable. ${ }^{25-27}$

Johnston and colleagues analyzed $\mathrm{K}^{+}$in arterial and venous blood samples taken from 50 patients during cardiac arrest; and ran them on an IL1640 BGA(Instrumentation Laboratory System, Lexington, Mass., USA). The venous sample was run for analysis via a pneumatic tube delivery system through a central Olympus AA(Beckman Coulter Inc., Miami, FL, USA). Johnston explained that the differences between the results are due to unidentified hemolysis. ${ }^{27}$

In their retrospective analysis, Açıkgöz and colleagues compared 118 patients with acute potassium elevations analyzed with an ABL 700 radiometer (Radiometer, Copenhagen, Denmark) with the Architect's c16000 AA (Abbott Diagnostics, Abbott Laboratories, ILL, USA). The mean difference between the two methods was $0.62 \pm 0.43$ $\mathrm{mmol} / \mathrm{L}(95 \%$ confidence intervals: 1.462 and -0.222$){ }^{28}$

\section{DISCUSSION}

There are several reasons for the differences observed in BGA and AA interchangeability studies for $\mathrm{Na}$ and $\mathrm{K}$ results.

The main reason for differences in the potassium value measured by the BGA device is that the hemolysis is not noticeable in the arterial specimen. Hawkins et al. reported that hemolysis is higher than predicted, and $33 \%$ of hypokalemic cases could not be detected with BGA. ${ }^{29}$ Venous samples are always centrifuged and then visually examined for hemolysis in routine laboratories. When hemolysis is detected, the sample is discarded, or no measurement is made for potassium. Meanwhile, the release of $\mathrm{K}$ from the platelets during coagulation may cause an increase in serum. ${ }^{30}$ 
The pneumatic tube system may lead to hemolysis, ${ }^{31}$ and potassium levels may differ in the central laboratory. ${ }^{18,27,32}$ Also; the difference between the time elapsed between sampling and analysis can influence the BGA and AA electrolyte measurements, especially $\mathrm{K} .{ }^{24}$

The use of different syringes or tubes containing the anticoagulant in sample preparations may be responsible for the preanalytical bias of the measured electrolytes in the BGA device. ${ }^{33-35}$ Dilution of the plasma volume of the sample with the use of conventional syringes washed with liquid heparin may cause the actual value of the BGAelectrolytes to be lower. ${ }^{34-37}$ In addition, heparin itself binds positively charged ions and lowers the value of the electrolytes measured. ${ }^{14,38}$

Studies have reported that when comparing BGA with an AA device, serum protein, and albumin levels are significantly important. ${ }^{17}$ The difference between the results was found to be correlated with serum albumin and total protein concentrations. ${ }^{9,22}$

It is important to detect small changes in the relative sodium concentrations in the follow-up of critical situations. ${ }^{38}$ Patients with symptomatic hyponatremia require slow correction, so patients' serum sodium levels should be monitored frequently. ${ }^{39}$ However, plasma protein levels may vary during treatment. In such cases, it is necessary to obtain the results from a single analyzer and minimize the analytical differences between the devices.

The accuracy and stability of the different calibrators used in each autoanalyzer are important for the reliability of the results, which can lead to differences in comparison studies. ${ }^{40}$

\section{Limitations}

For the measured variables, it is not possible to determine which electrolyte values (BGA vs. AA) are closer to the true value. Although external or internal quality-control studies are performed in routine laboratories; it does not show the real value of the sample.

\section{CONCLUSION}

It seems inappropriate to draw a conclusion about the interchangeability of different device results. Electrolyte levels should be regularly monitored, and the results of both measures should not be used interchangeably under the assumption that they are equivalent to each other.

\section{REFERENCES}

1. Fijorek K, Püsküllüo lu M, Tomaszewska D, Tomaszewski R, Glinka A, Polak S. Serum potassium, sodium and calcium levels in healthy individuals - literature review and data analysis. Folia Med Cracov 2014;54(1):53-70.

2. Weiss-Guillet EM, Takala J, Jakob SM. Diagnosis and manage- ment of electrolyte emergencies. Best Pract Res Clin Endocrinol Metab 2003;17(4):623-51.

3. Giordano M, Ciarambino T, Castellino P, Malatino L, Cataliotti $A$, Rinaldi $L$, et al. Seasonal variations of hyponatremia in the emergency department: Age-related changes. Am J Emerg Med 2017;35:749-752.

4. Bokemeyer A, Dziewas R, Wiendl H, Schwindt W, Bicsán $\mathrm{P}$, et al. Hyponatremia upon presentation to the emergency department - the need for urgent neuroimaging studies. Sci Rep 2017;7:1953.

5. Singer AJ, Thode HC Jr, Peacock WF. A retrospective study department of emergency potassium disturbances: severity, treatment, and outcomes. Clin Exp Emerg Med 2017;4:73-79.

6. Mousavi SA, Shahabi S, Mostafapour E, Purfakharan M, Fereshtehnejad SM, Amini J, et al. Comparison of the serum electrolyte levels among patients died and survived in the intensive care unit. Tanaffos 2012; 11: 36-42.

7. Scott MG, LeGrys VA, Klufts JC: Electrolytes and Blood Gases. Ch. 27. In Tietz Textbook of Clinical Chemistry. 4th edition. Edited by Burtis CA, Ashwood E, Bruns DE. Missouri USA: Elsevier; 2006:986.

8. Stove V, Slabbinck A, Vanoverschelde L, Hoste E, De Paepe $\mathrm{P}$, Delanghe J. How to solve the underestimated problem of overestimated sodium results in the hypoproteinemic patient. Crit Care Med 2016; 44:e83-8.

9. Nanda SK, Ray L, Dinakaran A. Agreement of arterial sodium and arterial potassium levels with venous sodium and venous potassium in patients admitted to intensive care unit. J Clin Diagn Res. 2015;9:28-30.

10. Budak YU, Huysal K, Polat M. Use of a blood gas analyzer and a laboratory autoanalyzer in routine practice to measure electrolytes in intensive care unit patients. BMC Anesthesiol. 2012; 3;12:17.

11. Ustundag-Budak $Y$, Huysal K. Application of sigma metrics and performance comparison Between two biochemistry analyser and a blood gas analyser for the determination of electrolytes. J Clin Diagn Res 2017; 11(2): BC06-BC09.

12. Kapoor D, Srivastava M, Singh P. Point of care blood gases with electrolytes and lactates in adult emergencies. Int J Crit Ill Inj Sci 2014;4(3):216-222.

13. Giavarina D. Understanding Bland Altman analysis. Biochem Med 2015 5;25(2):141-51.

14. Ehrmeyer SS, Laessig RH, Leinweber JE, Oryall JJ. 1990 Medicare/CLIA final rules for proficiency testing: minimum intralaboratory performance characteristics (CV and bias) needed to pass. Clin Chem 1990; 36: 1736-1740.

15. Yilmaz S, Uysal HB, Avcil M, Yilmaz M, Da lı B, Bakı M, et al. Comparison of different methods for measurement of electrolytes in patients admitted to the intensive care unit. Saudi Med J 2016;37(3):262-7.

16 King R, Campbell A. Performance of the radiometer OSM3 and ABL505 blood gas analysers for determination of sodium, potassium and haemoglobin concentrations. Anaesthesia 2000;55:65-9.

17. Allardet-Servent J, Lebsir M, Dubroca C, Fabrigoule M, Jordana S, Signouret T, et al. Point-of-Care versus central laboratory measurements of hemoglobin, hematocrit, glucose, bicarbonate and electrolytes: A prospective observational study in critically ill patients. PLoS One 2017; 10;12(1):

18. Chacko B, Peter JV, Patole S, Fleming JJ, Selvakumar R. Electrolytes assessed by point-of-care testing - Are the values 
comparable with results obtained from the central laboratory? Indian J Crit Care Med 2011;15(1):24-9

19. Auvet A, Espitalier F, Grammatico-Guillon L, Nay MA, Elaroussi D, Laffon M, et al. Preanalytical conditions of point-ofcare testing in the intensive care unit are decisive for analysis reliability. Ann Intensive Care.2016;6(1):57

20. Banerjee A, Mehrotra G.Role of correction factor in minimizing errors while calculating electrolyte values between Bloodgas analyzer and laboratory autoanalyzer: A comparative study. Indian J Crit Care Med. 2018;22(1):34-39.

21. Jose RJ, Preller J. Near-patient testing of potassium levels using arterial blood gas analysers: can we trust these results? Emerg Med J 2008;25(8):510-3.

22. Story DA, Morimatsu H, Egi M, Bellomo R. The effect of albumin concentration on plasma sodium and chloride measurements in critically ill patients. Anesth Analg 2007;104:8937.

23. Zhang JB, Lin J, Zhao XD. Analysis of bias in measurements of potassium, sodium and hemoglobin by an emergency department-based blood gas analyzer relative to hospital laboratory autoanalyzer results. PLoS One 2015 7;10(4):

24. Wongyingsinn $M$, Suksuriyayothin S. Use of rapid ABG analyzer in measurement of potassium concentration: does it agree with venous potassium concentration? J Med Assoc Thai 2009;92:925-9.

25. Morimatsu H, Rocktaschel J, Bellomo R, Uchino S, Goldsmith D, Gutteridge G. Comparison of point-of care versus central laboratory measurement of electrolyte concentrations on calculations of the anion gap and the strong ion difference. Anesthesiology 2003; 98: 1077 \pm 84

26. You JS, Park YS, Chung HS, Lee HS, Joo Y, Park JW, et al. Evaluating the utility of rapid point-of-care potassium testing for the early identification of hyperkalemia in patients with chronic kidney disease in the emergency department. Yonsei Med J 2014 ;55(5):1348-1353.

27. Johnston HL, Murphy R. Agreement between an arterial blood gas analyser and a venous blood analyser in the measurement of potassium in patients in cardiac arrest. Emerg Med J 2005;22(4):269-71.

28. Acikgoz SB, Genç AB, Sipahi S, Yildirim M, Cinemre B, Tamer A, et al. Agreement of serum potassium measured by blood gas and biochemistry analyzer in patients with moderate to severe hyperkalemia. Am J Emer Med 2016; 34 :794-797.

29. Hawkins R. Measurement of whole-blood potassium--is it clinically safe? Clin Chem 2003;49(12):2105-6.

30. Flegar-Mestric Z, Perkov S. Comparability of point-of-care whole-blood electrolyte and substrate testing using a stat profile critical care xpress analyzer and standard laboratory methods. Clin Chem Lab Med 2006;44:898-903.

31. Kara H, Bayir A, Ak A, Degirmenci S, Akinci M, Agacayak A, et al.Hemolysis associated with pneumatic tube system transport for blood samples. Pak J Med Sci 2014;30(1):50-8.

32. Petit M, Mine L, Pascreau T, Brouzes C, Majoux S, Borgel D, et al. Preanalytical influence of pneumatic tube delivery system on results of routine biochemistry and haematology analysis. Ann Biol Clin (Paris) 2017;75(6):703-712.

33. Hedberg P, Majava A, Kiviluoma K, Ohtonen P. Potential preanalytical errors in whole-blood analysis: effect of syringe sample volume on blood gas, electrolyte and lactate values. Scand J Clin Lab Invest 2009;69(5):585-91.

34. Lippi G, Salvagno GL, Montagnana M, Brocco G, Cesare Guidi G. Influence of the needle bore size used for collecting venous blood samples on routine clinical chemistry testing. Clin Chem Lab Med 2006;44(8):1009-14.

35. Chhapola V, Kumar S, Goyal P. Is liquid heparin comparable to dry balanced heparin for blood gas sampling in intensive care unit? Indian J Crit Care Med 2014;18(1):14-20.

36. Ancy J. Preventing preanalytical error in blood gas analysis (The blood gas laboratory) J Respir Care Sleep Med Spring 2012;30:64.

37. Jain A, Subhan I, Joshi M.Comparison of the point-of-care blood gas analyzer versus the laboratory auto-analyzer for the measurement of electrolytes. Int J Emerg Med 2009;2(2):117-20.

38. van Berkel $\mathrm{M}$, Scharnhorst V. Electrolyte-balanced heparin in blood gas syringes can introduce a significant bias in the measurement of positively charged electrolytes. Clin Chem Lab Med. 2011;49:249-52.

39. Loughrey CM , Hanna EV , McDonnell M , Archbold GP. Sodium measurement: effects of differing sampling and analytical methods. Ann Clin Biochem 2006; $43: 488$ - 93 .

40. Ge M, Zhao H, Yan Y, Zhang T, Zeng J, Zhou W, Wang Y, Meng Q, Zhang C. Performance of electrolyte measurements assessed by a trueness verification program. Clin Chem Lab Med 2016;54(8):1319-27. 